\title{
Use of EMLA cream as a topical anaesthetic before venepuncture procedures in field surveys: A practice that helps children, parents and health professionals
}

\author{
T P Gwetu, MB ChB, MPH; M K Chhagan, FCPaed, PhD \\ Department of Public Health, School of Clinical Medicine, College of Health Sciences, Nelson R Mandela School of Medicine, \\ University of KwaZulu-Natal, Durban, South Africa
}

Corresponding author: T P Gwetu (tgwetu@gmail.co.za)

\begin{abstract}
Background. Topical analgesia is becoming essential as the number of invasive screening procedures involving children rises steadily. Little is known about the frequency of these procedures, or about interventions to ease the pain.

Methods. We investigated the use of EMLA cream in 184 school-aged children in KwaZulu-Natal Province, South Africa. Another group of 20 children did not receive any local analgesia and was assessed as a control. Anticipatory anxiety, pain, adverse reactions and ease of procedure were assessed using a subjective visual analogue scale (VAS) pain score generated by the researcher and obtained from each child immediately after the procedure.

Results. The use of EMLA cream resulted in reduced pain and distress. The pain-relieving influence of EMLA was good ( $91.3 \%$ analgesic effect). Participants who received EMLA cream reported significantly lower VAS pain scores $(p=0.001)$. Pain scores generated by the researcher were also significantly lower in the EMLA group than in the control group $(p=0.000)$. No adverse reactions were observed, and the children could continue with other research activities during the application time and after the procedure. Parent or caregiver scores were in favour of EMLA cream.

Conclusion. EMLA cream was safe and effective for alleviating the pain associated with venepuncture in a fieldwork setting. We therefore believe that it merits a place in the routine premedication of children before phlebotomy and cannulation procedures in clinical settings, research studies and field surveys. Further research is recommended to assess whether EMLA cream can be used for immunisations.
\end{abstract}

S Afr Med J 2015;105(7):600-602. DOI:10.7196/SAMJnew.7797

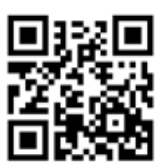

Children in healthcare settings endure painful procedures daily. Venepuncture of paediatric participants is also becoming increasingly common in many research studies and surveys. Several researchers have stated that venepuncture procedures are distressing to children and their families, and may affect the ease of performing the procedure, ${ }^{[1,2]}$ the success rate and the child-healthcare provider relationship. We could find no reports documenting the efficacy of EMLA cream in a field setting for the management of pain related to short procedures. Discomfort associated with these procedures continues to be inadequately managed.

Topical analgesia is becoming essential as the number of invasive screening procedures and health surveys involving children rises steadily. Children often do not understand why they are subjected to needle procedures such as phlebotomy. ${ }^{[2]}$ Little is known about the frequency of these invasive procedures, or about the use of interventions to ease the pain. A eutectic mixture of local anaesthetics (EMLA), which consists of $2.5 \%$ lidocaine and $2.5 \%$ prilocaine, is a topical analgesic that works by blocking pain transmission to the brain. Few studies have addressed whether use of a local anaesthetic influences venepuncture success and hence enrolment and retention rates of children in research studies and surveys. This study describes the efficacy of EMLA cream in preventing pain in school-aged children in a field setting. When carried out in a reassuring environment, this non-invasive means of delivering analgesia is expected to minimise distress during venepuncture procedures. The implications for practice and the benefits to both researchers and participants are expected to result in improved public health screening.

\section{Methods}

This study was an ancillary investigation in a larger longitudinal study measuring various health indicators and psychosocial needs of preschool and school-aged children. As far as possible, data collection was integrated into the usual services at the research site. One hundred and eighty-four consecutive children aged $6-8$ years attending for follow-up over a 4-month period were invited to join the current study. Another group of 20 children who did not receive any local analgesia was assessed as a control. EMLA application was limited to children requiring needle puncture of intact skin for phlebotomy procedures. The collected venous blood samples were used to assess the children's anaemia and iron status by laboratory assays for haemoglobin, serum ferritin and soluble transferrin receptor. These results are reported in another article. ${ }^{[3]}$

\section{Application}

The EMLA cream was applied at the research site before the phlebotomy procedure. The cream was applied to the skin surface under occlusion with a Tegaderm patch (3M). The dressing was placed at least 60 minutes before the phlebotomy procedure to allow for adequate local cutaneous absorption. A maximum of 120 minutes' application time was allowed to avoid significant systemic absorption of the lidocaine and prilocaine; $1-2 \mathrm{~cm}^{3}$ of cream was applied per $\mathrm{cm}^{2}$ of skin, up to a maximum of $10 \mathrm{~mL}$ on the same participant. During the application time the children continued with other scheduled research activities. The cream was removed and the skin disinfected before a venous blood sample was collected using a $22 \mathrm{G}$ Vacutainer needle. The participant was repeatedly asked about any skin or systemic reactions during and up to 2 hours after the application time. 


\section{Outcome assessment}

The primary outcome was analgesic efficacy for acute procedurerelated pain. Anticipatory anxiety, pain, adverse reactions and ease of procedure were assessed. A subjective visual analogue scale (VAS) pain score of $0-3$ was generated by the researcher and obtained through direct enquiry from each participant immediately after the phlebotomy procedure was completed. Pain responses were documented by the principal investigator (TPG) using subjective pain scores expressed through the VAS. A wry face was assessed as indicating mild pain, limb withdrawal as indicating moderate pain, and crying or resisting the procedure as indicating severe pain. The children were also asked about any pain they had experienced. The children's responses and the observations made by the clinician were both scored on a four-point verbal scale (pain-free -0 , mild pain -1 , moderate pain -2 , severe pain -3 ). The parent or caregiver observer ratings for the efficacy of EMLA cream in relieving pain were also considered.

\section{Statistical analysis}

The SPSS Statistics for Windows package, version 22.0 (IBM, USA), was used for data entry and analysis. Findings from the study were assessed with the variables characterised as either categorical or continuous. Categorical variables such as parental ratings of EMLA efficacy and anticipatory anxiety were analysed using a two-tailed Fisher's exact test. Continuous variables such as VAS provider and self-reported scores were assessed using Student's $t$-test and graphical plots. The study reported on the effects of EMLA with regard to the mean difference in pain, anxiety and ease of procedure during phlebotomy between the group that received EMLA and the control group with no intervention, relative risk (RR), and whether the effect on pain reduction was significant or non-significant.

\section{Ethical considerations}

This research received ethical clearance from the official review board of the University of KwaZulu-Natal Biomedical Research Ethics Committee. Informed consent was obtained from the children's caregivers before they were included in the study.

\section{Results}

One hundred and eighty-four children participated in the study. The participants' ages ranged from 72 to 102 months (mean 83.65, standard deviation 7.32). There were 109 males (59.2\%) and fewer females $(n=75,40.8 \%)$. The mean application time was 72 minutes (range 60 - 104).

\section{Anticipatory anxiety}

The children were individually counselled before application of the cream, and told that it would help reduce the pain. This, together with a reassuring environment, resulted in the children co-operating with the phlebotomy procedure; $24 / 184(13.0 \%)$ were noted to be mildly anxious, although they allowed the phlebotomy procedure to continue. The children in the control group were generally anxious $(12 / 20,60.0 \%)$ and showed some distress despite the reassuring environment.

\section{Pain and distress}

EMLA cream was effective in reducing pain during phlebotomy in $91.3 \%$ of participants compared with $25.0 \%$ in the control group. Of children who underwent procedures conducted with EMLA, only $3 / 184(1.6 \%)$ complained of moderate pain and $13 / 184$ (7.1\%) of mild discomfort. The rest $(n=168,91.3 \%)$ were without pain. No child reported severe pain. The researcher's pain scores were significantly lower in the EMLA group than in the control group $(p=0.000)$. The participants who received EMLA also selfreported significantly lower pain scores $(p=0.001)$. With regard to anaesthetic efficacy, EMLA significantly reduced the risk of pain compared with the control group (RR $0.11 ; 95 \%$ confidence interval (CI) $0.05-0.27$ ), self-reporting of pain (RR 0.12 ; $95 \%$ CI 0.04 $0.24)$, and observations by the investigators of responses indicating pain (RR 0.13; 95\% CI 0.02 - 0.22).

\section{Adverse reactions}

EMLA cream was well tolerated and non-toxic in this study population. No adverse dermatological or systemic reactions were noted, and no itching or local reactions were observed. The children were able to continue with other scheduled research activities during the application time and also after the phlebotomy procedure.

\section{Ease of procedure}

Of phlebotomies with EMLA, 172/184 (93.5\%) were graded as 'easy' compared with $12 / 20(60.0 \%)$ in the control group. Easier needle insertion was noted with EMLA cream, and this was statistically significant $(R=0.417 ; p=0.000)$.

\section{Parent or caregiver ratings}

The ratings of EMLA cream efficacy by parent or caregiver observer scales were in favour of the cream. They felt that EMLA cream was effective in decreasing the distress associated with the phlebotomy procedure in 180/184 children (97.8\%).

\section{Discussion}

This study found the use of EMLA cream to be an effective strategy for managing acute procedure-related pain in children participating in field surveys. EMLA also improved the ease of the procedure. The reduced trouble and effort associated with restraining a struggling child has been reported by other researchers. ${ }^{[4]}$ The $33.5 \%$ increase in ease of drawing blood when EMLA was used (93.5\% for the EMLA group minus $60.0 \%$ for the control group) suggests that topical analgesia offers a substantial benefit. The analgesic effect of EMLA for this study population was $91.3 \%$, comparable to other studies conducted in outpatient settings. ${ }^{[5,6]}$ Errors that may cause inadequate analgesia include inappropriate application, a short waiting time, using a small volume of cream, and poor occlusion strategies such as a loose Tegaderm dressing. ${ }^{[1,2]}$ These errors were minimised in this study by controlling the application time to between 1 and 2 hours, measuring the volume of cream used, and having trained medical personnel apply the cream and dressing.

EMLA was well tolerated and non-toxic. No participants developed side-effects, although this could be due to the small sample size and/ or the short follow-up period. Although EMLA is generally reported to be non-toxic after infancy, local adverse outcomes have been described by other researchers and include erythema, oedema, allergic contact dermatitis and hyperpigmentation. ${ }^{[7]}$ Seizures and respiratory depression were described in one case report. ${ }^{[8]}$ Methaemoglobinaemia was the most dangerous and potentially fatal complication reported in neonates. ${ }^{[9]}$

The limitations of EMLA use observed in this study included the need to wait for at least 60 minutes from application of the cream to the venepuncture procedure. The considerable waiting time required before an adequate analgesic effect could be overcome by allowing capable children and their caregivers to participate in the application after being instructed in the proper use of the cream. ${ }^{[10]}$ The application of EMLA could also be scheduled earlier, while the child participates in other research activities or in the 
waiting area. It was not possible to distinguish between problematic venepunctures that necessitated numerous needle insertions and anticipatory anxiety resulting in the child struggling even when pain relief was adequate. Anticipatory anxiety can be expected to lessen with widespread use of pharmacological and nonpharmacological strategies to reduce needle phobias. ${ }^{[11]}$ If adequate pain relief is provided, children can be expected to become less fearful of needle-related procedures. The cost-effectiveness of the cream may be an issue if it is used in on a large scale, and needs to be weighed against the benefits of a stress-free, easier and safer venepuncture procedure.

Needle phobia is a recognised medical disorder, affecting approximately $10 \%$ of the world population and resulting in fear and avoidance behaviour. ${ }^{[12]}$ In the current study, $13.0 \%$ of the children who received EMLA cream were anxious about the procedure v. $60.0 \%$ in the control group. In an era when health of children in underdeveloped countries is being prioritised, with large-scale screening and intervention initiatives, avoidance of healthcare in order to avoid needle experience would be unfortunate. ${ }^{[12]}$ Many screening tests and interventions rely on the hypodermic needle. Health professionals need to be mindful of the fact that needle phobia, although infrequent, may lead to avoidance of health initiatives. The use of topical analgesia in children should be prioritised, and health professionals need to be aware of available options to prevent the pain associated with needle procedures.

The use of EMLA cream can be expected to result in safer and more effective venepuncture procedures in children by lessening the discomfort experienced by the child and making it easier to perform these procedures. Numerous other non-invasive topical anaesthetic options are available that can be measured against EMLA. Welldesigned clinical trials are necessary in order to assess EMLA further with regard to relative efficacy, safety and feasibility in children in a large-scale field setting in national surveys and also for childhood immunisations. Further studies, such as case-control studies, are also necessary to determine whether a waiting time of $<60$ minutes can be used without compromising the efficacy of the cream.

\section{Conclusion}

Use of EMLA cream can reduce pain, make venepuncture easier, and improve the therapeutic relationship with health professionals Further research is recommended to assess whether EMLA can be used for childhood immunisations.

\section{References}

1. Chen BK, Cunningham BB. Topical anaesthetics in children: Agents and techniques that equally comfort patients, parents, and clinicians. Current Opinion in Paediatrics 2001;13(4):324-330. [http:// dx.doi.org/10.1097/00008480-200108000-00007]

2. Rogers TL, Ostrow CL. The use of EMLA cream to decrease venepuncture pain in children. J Pediat Nurs 2004;19(1):33-39. [http://dx.doi.org/10.1016/j.pedn.2003.09.005]

3. Gwetu TP, Chhagan MK. Evaluation of the diagnostic accuracy of the HaemoCue device for detecting anaemia in healthy school-aged children in KwaZulu-Natal, South Africa. S Afr Med J 2015;105(7):596599. [http://dx.doi.org/10.7196/SAM]new.7919]

4. Choy L, Collier J, Watson AR. Comparison of lidocaine-prilocaine cream and amethocaine gel for local analgesia before venepuncture in children. Acta Paediatr 1999;88(9):961-964. [http://dx.doi. org/10.1111/j.1651-2227.1999.tb00190.x]

5. Hellgren U, Kihamia CM, Premji Z, Danielson K. Local anaesthetic cream for the alleviation of pain during venepuncture in Tanzanian schoolchildren. Br J Clin Pharmacol 1989;28(2):205-206

6. Young SS, Schwartz R, Sheridan MI. EMLA cream as a topical anaesthetic before office phlebotomy 6oung SS, Schwartz R, Sheridan M). EMLA cream as a topical anaesthetic before office phlebotom in Children. South Med J 1996,8(12):1184-1187. [htp.//x.doi.org/10.10970007611-199612000-

7. Godwin Y, Brotherston M. Hyperpigmentation following the use of EMLA cream. Br J Plast Surg 2001;54(1):82-83. [http://dx.doi.org/10.1054/bjps.2000.3466]

8. Rincon E, Baker RL, Iglesias AJ, et al. CNS toxicity after topical application of EMLA cream on a toddler with molluscum contagiosum. Pediatr Emerg Care 2000;16(4):252-254. [http://dx.doi. org/10.1097/00006565-200008000-00009

9. Essink-Tebbes CM, Wuis EW, Liem KD, et al. Safety of lidocaine-prilocaine cream application fou times a day in premature neonates: A pilot study. Eur J Pediatr 1999;158(5):421-423. [http://dx.doi. org $/ 10.1007 / 5004310051106]$

10. Koh JL, Fanurik D, Stoner PD, et al. Efficacy of parental application of eutectic mixture of local anesthetics for intravenous insertion. Pediatrics 1999;103:e79. [http://dx.doi.org/10.1542/ peds.103.6. 79$]$

11. Sokolowski CJ, Giovannitti JA, Boynes SG. Needle phobia: Etiology, adverse consequences, and patient management. Dent Clin North Am 2010;54(4):731-744. [http://dx.doi.org/10.1016/..cden.2010.06.012]

12. Hamilton JG. Needle phobia: A neglected diagnosis. J Fam Pract 1995:41(2):169-175. 